\title{
Endoscopic third ventriculostomy with choroid plexus cauterization for the treatment of infantile hydrocephalus in Haiti
}

\author{
Ashish H. Shah, MD, ${ }^{1}$ Yudy LaFortune, MD, ${ }^{2}$ George M. Ibrahim, MD, PhD, ${ }^{3}$ lahn Cajigas, MD, PhD, ${ }^{1}$ \\ Michael Ragheb, MSPH, ${ }^{1,6}$ Stephanie H. Chen, MD, ${ }^{1}$ Ernest J. Barthélemy, MD, MA, MPH, ${ }^{4,5}$ \\ Ariel Henry, MD, ${ }^{2}$ and John Ragheb, MD ${ }^{4,6}$
}

1Department of Neurological Surgery, University of Miami, Florida; ${ }^{2}$ Department of Neurological Surgery, Hospital Bernard-Mevs/ Project Medishare, Port-au-Prince, Haiti; ${ }^{3}$ Division of Neurosurgery, Hospital for Sick Children, University of Toronto, Department of Surgery, Toronto, Ontario, Canada; ${ }^{4}$ Department of Neurosurgery, Mount Sinai Health System, New York, New York; ${ }^{5}$ Program in Global Surgery and Social Change, Harvard Medical School, Boston, Massachusetts; and ${ }^{6}$ Division of Neurosurgery, Nicklaus Children's Hospital, Miami, Florida

OBJECTIVE Untreated hydrocephalus poses a significant health risk to children in the developing world. In response to this risk, global neurosurgical efforts have increasingly focused on endoscopic third ventriculostomy with choroid plexus cauterization (ETVICPC) in the management of infantile hydrocephalus in low- and middle-income countries (LMICs). Here, the authors report their experience with ETVICPC at the Hospital Bernard-Mevs/Project Medishare (HBMPM) in Port-au-Prince, Haiti.

METHODS The authors conducted a retrospective review of a series of consecutive children who had undergone ETVI CPC for hydrocephalus over a 1-year period at HBMPM. The primary outcome of interest was time to ETVICPC failure. Univariate and multivariate analyses using a Cox proportional hazards regression were performed to identify preoperative factors that were associated with outcomes.

RESULTS Of the 82 children who underwent ETVICPC, 52.2\% remained shunt free at the last follow-up (mean 6.4 months). On univariate analysis, the ETV success score (ETVSS; $p=0.002)$, success of the attempted ETV $(p=0.018)$, and bilateral CPC ( $p=0.045)$ were associated with shunt freedom. In the multivariate models, a lower ETVSS was independently associated with a poor outcome (HR 0.072, 95\% Cl 0.016-0.32, p < 0.001). Two children $(2.4 \%)$ died of postoperative seizures.

CONCLUSIONS As in other LMICs, ETVICPC is an effective treatment for hydrocephalus in children in Haiti, with a low but significant risk profile. Larger multinational prospective databases may further elucidate the ideal candidate for ETVI $\mathrm{CPC}$ in resource-poor settings.

https://thejns.org/doi/abs/10.3171/2019.10.PEDS19433

KEYWORDS Haiti; hydrocephalus; endoscopic; ventriculostomy; cauterization

$\mathrm{T}$ HERE is significant global disparity in the incidence of hydrocephalus, with a 20 -fold higher case volume in low- and middle-income countries (LMICs) compared to that in high-income countries. ${ }^{4,21}$ In Haiti, the actual incidence of hydrocephalus is unknown, but it is estimated that more than 600 new cases of hydrocephalus occur annually., ${ }^{4,614,21}$ Several factors may contribute to these disparities, including limited access to prenatal or perinatal care, high rates of neonatal infections, and limited healthcare infrastructures. ${ }^{1}$
The care of children with hydrocephalus is also limited by lack of access to neurosurgical resources. With only a few neurosurgeons with limited resources in a country of over 11 million people (yielding a provider ratio of less than 0.04 neurosurgeons per 100,000 persons), direct neurosurgical care in Haiti is often delayed or impossible. Moreover, surgical treatment options have historically remained limited to a surgeon's technical capacity and the availability of surgical equipment. While ventriculoperitoneal shunting (VPS) is the most common treatment strat-

ABBREVIATIONS CPC = choroid plexus cauterization; ETV = endoscopic third ventriculostomy; ETVSS = ETV Success Score; HBMPM = Hospital Bernard-Mevs/Project Medishare; LMICs = low- and middle-income countries; PIH = postinfectious hydrocephalus; VPS = ventriculoperitoneal shunting.

SUBMITTED August 5, 2019. ACCEPTED October 16, 2019.

INCLUDE WHEN CITING Published online January 10, 2020; DOI: 10.3171/2019.10.PEDS19433. 
egy for infantile hydrocephalus, limited access to shunts and challenges in effectively troubleshooting shunt complications have curbed the procedure's utility in LMICs. Complication rates for VPS can exceed $30 \%$, including malfunction or migration, over-shunting, infection, and wound breakdown. ${ }^{2,13,17}$

As in other developing countries, namely Uganda, endoscopic third ventriculostomy with choroid plexus cauterization (ETV/CPC) has been offered as a durable, safe alternative to VPS. With its similar neurodevelopmental outcomes and reduced shunting rates compared to those of VPS, ETV/CPC has become a mainstay primary treatment of infantile hydrocephalus in Africa. ${ }^{11}$ However, since the majority of the literature advocating the use of ETV/CPC arises from the Uganda experience, little is known about the generalizability of these results to other LMICs. With the recent establishment of a neurosurgical residency program in Haiti, ETV/CPC has become a primary surgical treatment option for pediatric hydrocephalus, ${ }^{16}$ which was previously challenging. ${ }^{7}$ Here, we report on our initial consecutive series of ETV/CPC for infantile hydrocephalus over a 1-year period in one of the LMICs within the Western Hemisphere.

\section{Methods}

After receiving institutional review board approval from the University of Miami, we performed a retrospective review of a prospectively maintained database of infants with hydrocephalus (RedCap). The goal of this registry is to prospectively collect data and assess prognosticators for hydrocephalic infants. The majority of patients in our initial cohort were infants (age $<1$ year) who had been treated at one tertiary care referral center, Hospital Bernard-Mevs/Project Medishare (HBMPM), in Port-au-Prince, Haiti, over a 1-year time span (2016-2017). The diagnosis of hydrocephalus was confirmed by standard clinical and radiological criteria (elevated head circumference, progressive macrocephaly, bulging fontanelle, upward gaze palsy, severe ventriculomegaly on screening CT) ${ }^{19}$ All etiologies of hydrocephalus were included in our study (postinfectious hydrocephalus [PIH], aqueductal stenosis, myelomeningocele, premature hemorrhagic hydrocephalus, Dandy-Walker malformation, communicating hydrocephalus, and other). PIH was determined by the following criteria, which have been outlined by Warf: no previous history of hydrocephalus, a history of febrile illness with or without seizures prior to the development of hydrocephalus, and endoscopic and radiographic evidence of prior infection (subarachnoid scarring, loculations, debris, calcifications, etc.). ${ }^{19}$ None of the children, except one, had received any prior treatment for hydrocephalus. All patients with hydrocephalus during this time period were all primarily offered ETV/CPC. The ETV/CPC was attempted, as described previously, via a rigid endoscope, and CPC was performed bilaterally when feasible. ${ }^{3,24}$

Patient demographics (gestational age, region, sex) and relevant clinicoradiological parameters (head circumference and etiology) as well as intraoperative findings were recorded. An ETV Success Score (ETVSS) was calculated (scores 0-90) as previously described in the literature. ${ }^{9}$ The primary outcome was failure of ETV/CPC, which was based on the need for an additional CSF diversion procedure (VPS or repeat ETV) during the follow-up period. Failure of ETV was determined primarily by the treating surgeon based on clinical examination and imaging. Patients who were lost to follow-up were contacted by our HBMPM team; patients who were lost to follow-up were censored for survival analysis.

\section{Statistical Analysis}

All descriptive statistics are presented as means or medians and standard deviations or proportions. The primary outcome of interest was time to ETV/CPC failure. Univariate Cox proportional hazards regression was performed to identify associations between patient covariates and time to event. Covariates with $\mathrm{p}<0.20$ on univariate analysis were included in the multivariate model. In addition, infectious etiology was included in the multivariate model because of the known negative effects of infectious etiology on failure-free survival of ETV/CPC. ${ }^{19,20,22,23}$ All final models were deemed significant at $p<0.05$. Statistical analysis was performed using functions programmed in $\mathrm{R}$ statistical software ( $\mathrm{R}$ Foundation for Statistical Computing).

\section{Results}

Over the 1-year time period, 82 children with hydrocephalus who had undergone attempted ETV/CPC for hydrocephalus were identified at HBMPM (82 procedures). The median age of the children undergoing ETV/CPC was 7.5 months, and most of the patients were male $(n=$ 49 [59.8\%]). The predominant etiologies of hydrocephalus were aqueductal stenosis $(n=24$ [29.3\%]) and PIH $(n=22$ [26.8\%]; Table 1). The median ETVSS was 50. Successful ETV was performed in $86.6 \%$ of patients $(n=71)$, with evidence of ostomy pulsation in $79.2 \%(n=65)$. ETV was aborted in the remaining patients because of poor visualization (5 cases) or intraoperative hemorrhage (6 cases). Bilateral, unilateral, or no CPC was performed in $49(59.8 \%)$, 20 (24.4\%), and $13(15.9 \%)$ patients, respectively (Table 2). Bilateral CPC was not performed in infants with minimal or no choroid plexus. Prepontine scarring was noted in $40.2 \%$ of patients $(n=33)$.

Of this cohort, $52.2 \%$ remained shunt free at the last follow-up (mean 6.4 months); 5 patients were lost to follow-up (Fig. 1). Perioperative death due to seizures was noted in $2.4 \%$ of cases that suffered from intraventricular hemorrhage during CPC. Superficial wound issues (infection and CSF leakage) were noted in $4.9 \%$ of cases $(n=4)$. On univariate analysis, the ETVSS $(\mathrm{p}=0.002)$, success of the ETV $(p=0.018)$, and bilateral CPC $(p=0.045)$ were associated with shunt-free survival (Fig. 2). In the multivariate models, a moderate ETVSS (50-70; HR 0.073, 95\% CI 0.019-0.28, p < 0.001), high ETVSS ( $\geq 80$; HR $0.084,95 \%$ CI $0.018-0.39, p=0.002)$, and PIH etiology (HR $0.058,95 \%$ CI $0.012-0.29, \mathrm{p}<0.001$ ) were independently associated with longer shunt-free survival (Fig. 3).

\section{Discussion}

The use of ETV/CPC as a primary treatment for pe- 
TABLE 1. Preoperative characteristics of 82 children treated with ETVICPC at HBMPM

\begin{tabular}{lc}
\hline \multicolumn{1}{c}{ Characteristic } & No. \\
\hline Median age in mos (IQR) & $7.5(11)$ \\
\hline Sex, no. (\%) & $49(59.8)$ \\
\hline Male & $33(40.2)$ \\
\hline Female & $3(3.7)$ \\
\hline Age at surgery in mos, no. (\%) & $21(25.6)$ \\
\hline$<2$ & $28(34.1)$ \\
\hline $2-6$ & $30(36.6)$ \\
\hline $6-12$ & $1(1.2)$ \\
\hline$>12$ & $24(29.3)$ \\
\hline Etiology, no. (\%) & $4(4.9)$ \\
\hline IVH of prematurity & $11(13.4)$ \\
\hline Aqueductal stenosis & $13(15.9)$ \\
\hline Myelomeningocele & $22(26.8)$ \\
\hline Dandy-Walker & $7(8.5)$ \\
\hline Communicating hydrocephalus & $1(1.2)$ \\
\hline PIH & $50(32.5)$ \\
\hline Other & $5(3)$ \\
\hline Prior shunt & 6.4 \\
\hline Median ETVSS (IQR) & $1 V H(1962)$ \\
\hline Median CCHU ETVSS (IQR) & \\
\hline Mean FU in mos & \\
\hline
\end{tabular}

CCHU = CURE Children's Hospital of Uganda; FU = follow-up; IVH = intraventricular hemorrhage.

diatric hydrocephalus in LMICs was largely popularized in Uganda almost 20 years ago. The primary goal was to avoid long-term complications associated with VPS in resource-poor settings despite the higher short-term failure rates (first 6 months) with ETV/CPC. Over the years, the procedure has been validated in randomized controlled trials that confirm equivalent short-term neurodevelopmental outcomes for ETV/CPC and VPS. ${ }^{11,13,18,20}$ Despite these results, translation of this practice beyond Africa and specifically to Haiti has remained challenging. This is largely attributable to the limited infrastructure and minimal neurosurgical capacity in Haiti. To address this, the Project Medishare Subspecialty Surgery program has worked for the last 2 decades to provide sustainable pediatric neurosurgical care in Haiti. Since its inception, the program has treated over 1000 children with hydrocephalus and has recently gained accreditation for the nation's first neurosurgery training program..$^{16}$ The program is primarily based out of Hospital Bernard-Mevs in Port-auPrince, the nation's only trauma center with a partnership with the State University of Haiti medical school. Through a hybrid apprenticeship model combining a visiting professorship with local neurosurgical mentorship, the fellowship is the first local training program of its kind to successfully train Haitian neurosurgeons to autonomously perform ETV/CPC for pediatric hydrocephalus. ${ }^{16}$ Our results illustrate the preliminary results of a patient cohort treated by the country's first neurosurgery resident (Y.L.) over a 1-year time frame.
TABLE 2. Intraoperative variables in 82 patients with attempted ETV/CPC in Haiti

\begin{tabular}{cc}
\hline Characteristic & No. (\%) \\
\hline ETV performed & $71(86.6)$ \\
\hline Third ventricular pulsation & $65(79.2)$ \\
\hline CPC performed & $69(84.2)$ \\
\hline Extent of CPC & $13(15.9)$ \\
\hline None & $20(24.4)$ \\
\hline Unilat & $49(59.8)$ \\
\hline Bilat & \\
\hline Intraop IVH & $75(91.5)$ \\
\hline None & $4(4.9)$ \\
\hline Mild & $2(2.4)$ \\
\hline Moderate & $1(1.2)$ \\
\hline Severe & $33(40.2)$ \\
\hline Prepontine scarring &
\end{tabular}

The results of our study demonstrate that ETV/CPC is a viable and safe treatment option for pediatric hydrocephalus, with shunt freedom rates comparable to those at other centers. Previously, ETV/CPC success rates at other sites have been in the range from $40 \%$ to $70 \%$. $^{5,11,15,22,23}$ Our rates are slightly lower than other established international pediatric neurosurgery training sites $(75 \%)$, which may reflect several limitations in the management of hydrocephalus surgery in Haiti. Primarily, our series highlights outcomes for an intent-to-treat analysis, that is, patients with attempted ETV/CPC. Although most patients underwent ETV (86.6\%), the procedure was not feasible in select patients because of unfavorable anatomy, impaired visualization, or intraoperative hemorrhage. If the patients who did not undergo ETV are excluded, the ETV/CPC success rate remains $69 \%$ at the last follow-up. Additionally, our cohort has an overwhelmingly high percentage of PIH, which may affect the efficacy of ETV/CPC. Historically, PIH has higher rates of failure after ETV/CPC than obstructive etiologies, which is likely attributable to poor intraoperative visualization, septations, and prepontine arachnoid scarring. ${ }^{8-10,12,25}$ In our series, however, we found that non-PIH infants had a worse outcome than PIH infants. This difference may be attributable to poor ETV success in non-PIH infants with severe hydrocephalus and myelomeningocele or Dandy-Walker malformation. Nevertheless, we plan to revisit this finding as we accrue a larger number of patients over the next few years.

\section{Complications}

From our initial series of ETV/CPC, there were 2 early postoperative deaths due to seizures in patients who had suffered from intraventricular hemorrhage during surgery. These seizures may be secondary to the corticotomy to introduce the endoscope or to electrolyte imbalances from the use of normal saline irrigation during endoscopic lavage. The current practice in Haiti is to use lactated ringers instead of normal saline to best recapitulate the normal CSF electrolyte balance. Additionally, 4 patients suffered from superficial wound issues that were managed with a 

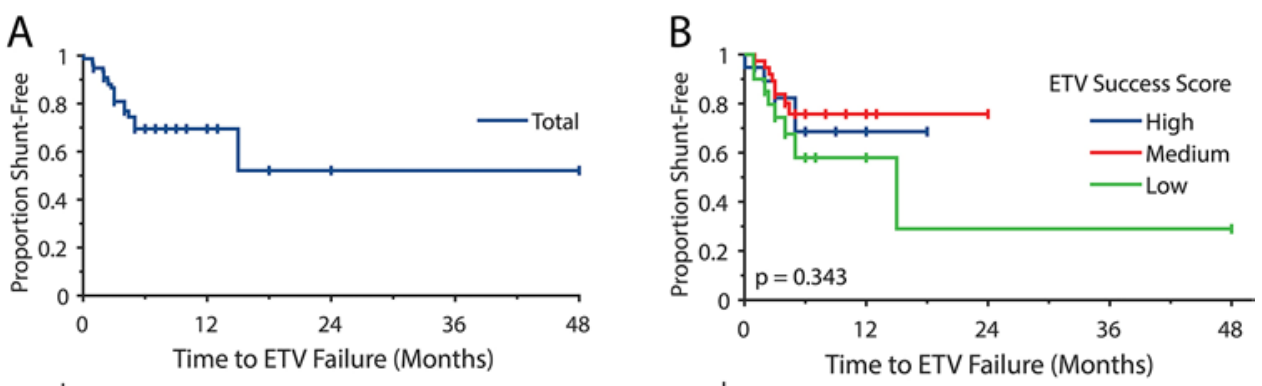

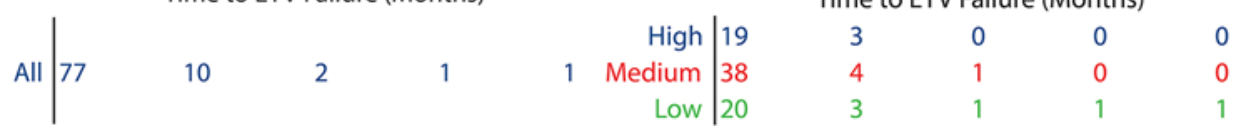

FIG. 1. Time to VPS after attempted ETVICPC. A: Whole cohort. B: Cohort stratified based on ETVSS (high > 80, medium 50-80, low $<50)$. Figure is available in color online only.

short course of oral antibiotics and wound reinforcement. Cases with persistent CSF leakage were referred for VPS and were considered treatment failures.

\section{Study Limitations}

Our study is limited by the inherent nature of retrospective reviews including selection bias and lack of long-term follow-up. Since our inclusion criteria were mostly subjective, there may be a selection bias since the inclusion of hydrocephalic infants was mostly determined by clinical and radiological criteria judged by the treating neurosurgeon. Although ETV/CPC is known for early treatment failure (first 6 months), our study was not designed to assess long-term outcomes following endoscopic treatment.
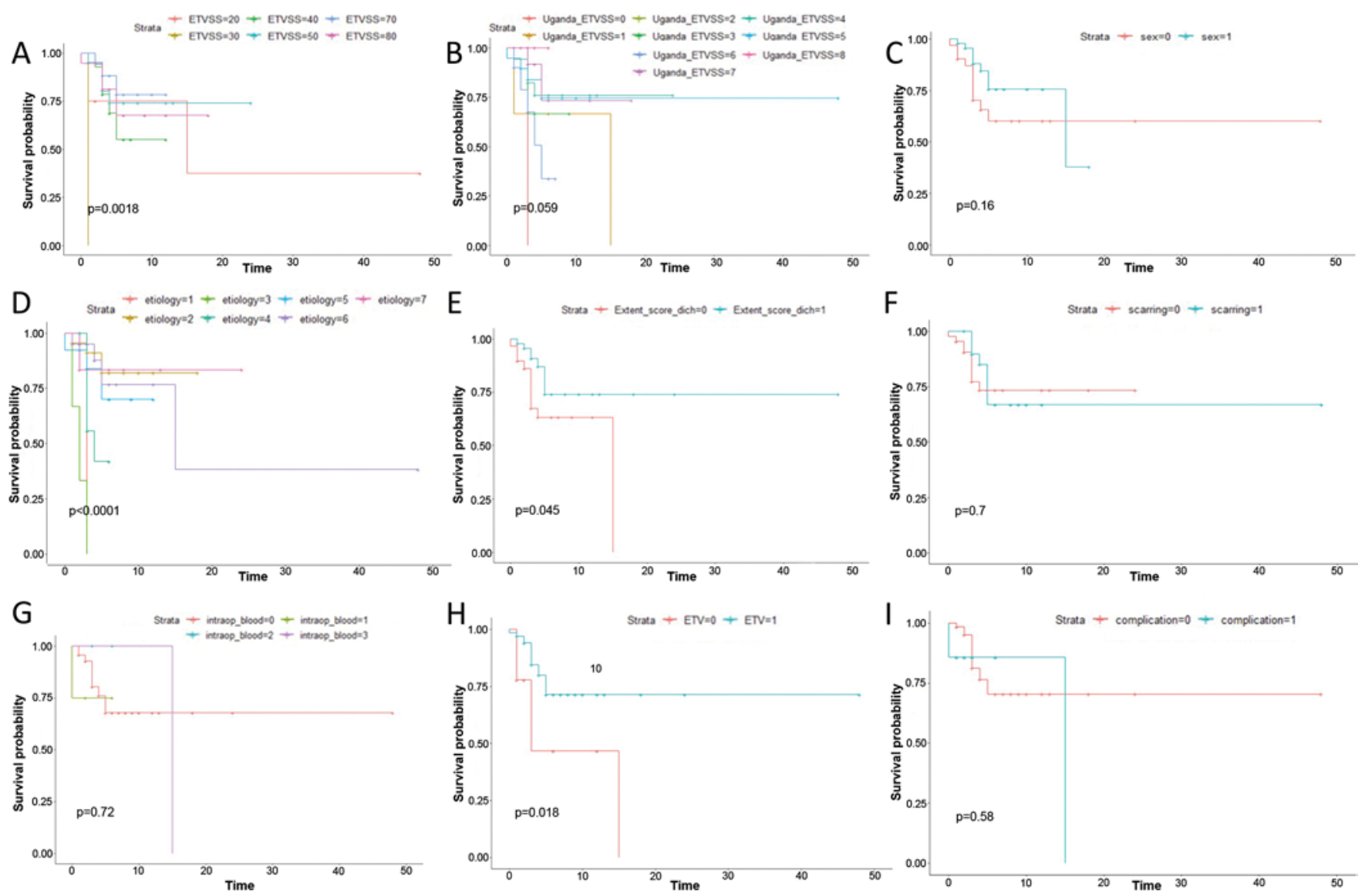

FIG. 2. Kaplan-Meier univariate analysis for predictors of shunt freedom stratified by ETVSS (A); Uganda CURE ETVSS (B); sex: $0=$ female, 1 = male $(\mathbf{C})$; etiology: intraventricular hemorrhage of prematurity $=1$, congenital aqueductal stenosis $=2$, myelomeningocele $=3$, Dandy-Walker malformation $=4$, congenital communicating hydrocephalus $=5, \mathrm{PIH}=6$, other $=7$ (D); bilateral $\mathrm{CPC}$ : $0=$ no, $1=$ yes $(E)$; presence of prepontine scarring: $0=$ no, $1=$ yes $(F)$; intraoperative bleeding: $0=$ no, $1=$ mild, $2=$ moderate,

$3=$ severe (G); ETV successfully performed: $0=$ no, $1=$ yes $(\mathbf{H})$; and presence of postoperative complications: $0=$ no, $1=$ yes $(\mathbf{I})$. Figure is available in color online only. 


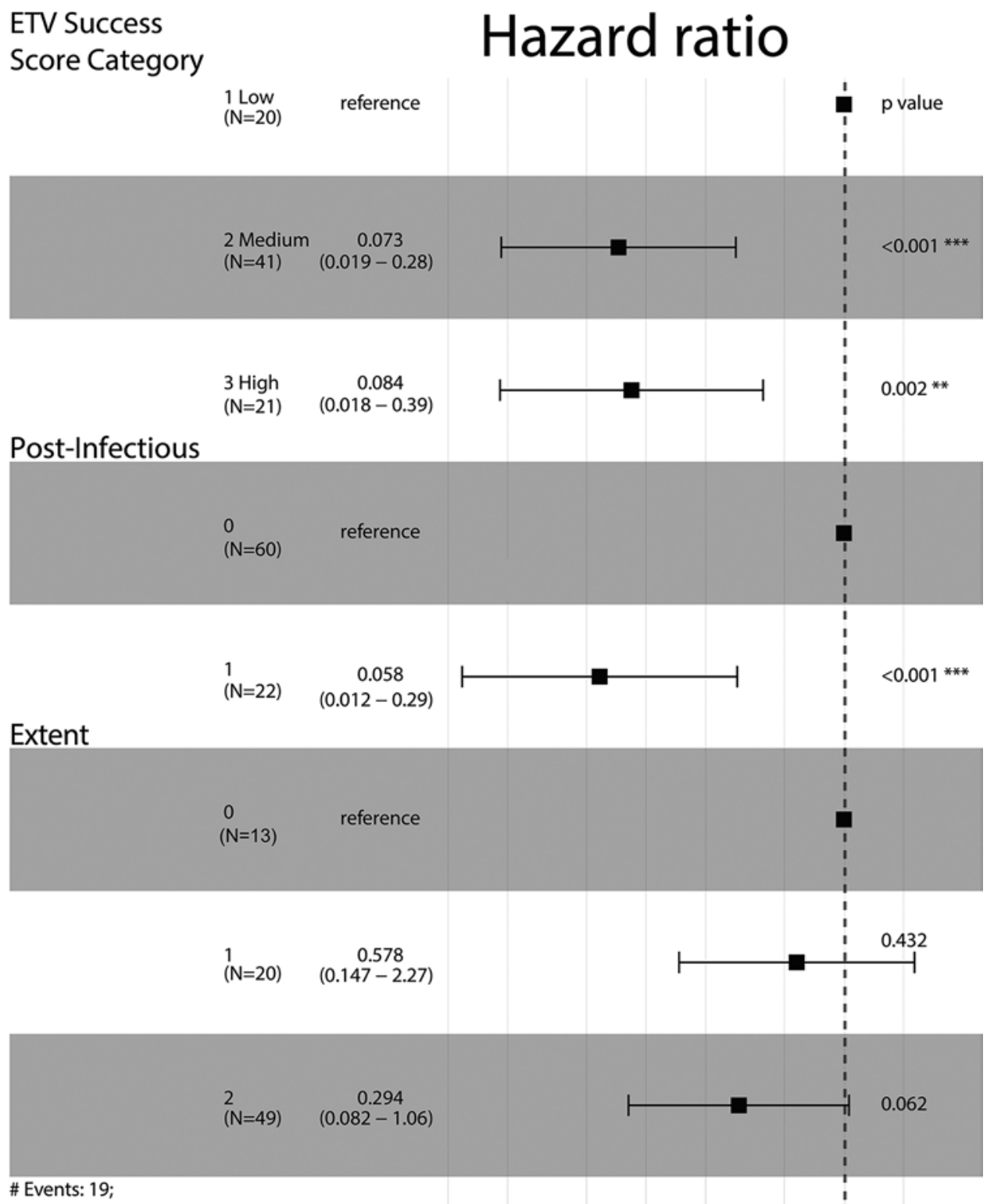

FIG. 3. Multivariate Cox proportional hazards regression for predictors of shunt freedom. Extent of CPC: $0=$ none, $1=$ unilateral, 2 = bilateral; presence of $\mathrm{PIH}=1$.

Additionally, the heterogeneity of the pathology limits extrapolation of our data to other centers; however, our data serve as evidence of the feasibility of a sustainable hydrocephalus program in a severely resource-limited setting.

\section{Future Directions}

With an established infrastructure and preliminary data assessing the efficacy of ETV/CPC in Haiti, systematic prospective studies can be performed to evaluate the utility of ETV/CPC. Specifically, it remains paramount to assess preoperative and postoperative neurodevelopmental quotients to determine long-term developmental trajectories after endoscopy. Additionally, understanding and identifying etiological factors of hydrocephalus would also be instrumental in reducing the incidence of hydrocephalus and disability in children in Haiti. A larger database including demographic and epidemiological information may better direct potential preventative strategies in the community (i.e., maternal hygiene, immunizations, prenatal vitamins, etc.). Ultimately, expansion of the residency program will usher in a more sustainable hydrocephalus program that will inevitably reduce the burden of disease.

\section{Conclusions}

As in other LMICs, ETV/CPC remains a viable, efficacious procedure for reducing the need for VPS in Haiti, with a low but significant risk profile. Given the limited access to neurosurgical care, ETV/CPC is likely to remain a mainstay in the treatment of infant hydrocephalus in this nation. Among all etiologies, PIH remains one of the leading causes of hydrocephalus in Haiti, albeit with good outcomes after attempted ETV/CPC (compared to non-PIH 
infants). With recent neurosurgical capacity-building efforts, larger multinational prospective databases may elucidate the role of ETV/CPC in resource-poor settings.

\section{Acknowledgments}

We dedicate this work in memory of Dr. Sanjiv Bhatia, who worked with the Project Medishare Hydrocephalus program since inception in 2003. His dedication to the children of Haiti and to teaching the future neurosurgeons of Haiti has changed the lives of hundreds of children and will have an enduring effect on healthcare in Haiti.

\section{References}

1. Boulos A, Rand K, Johnson JA, Gautier J, Koster M: Neonatal sepsis in Haiti. J Trop Pediatr 63:70-73, 2017

2. Browd SR, Ragel BT, Gottfried ON, Kestle JR: Failure of cerebrospinal fluid shunts: part I: Obstruction and mechanical failure. Pediatr Neurol 34:83-92, 2006

3. Chamiraju P, Bhatia S, Sandberg DI, Ragheb J: Endoscopic third ventriculostomy and choroid plexus cauterization in posthemorrhagic hydrocephalus of prematurity. J Neurosurg Pediatr 13:433-439, 2014

4. Dewan MC, Rattani A, Mekary R, Glancz LJ, Yunusa I, Baticulon RE, et al: Global hydrocephalus epidemiology and incidence: systematic review and meta-analysis. J Neurosurg 130:1065-1079, 2019

5. Drake JM: Endoscopic third ventriculostomy in pediatric patients: the Canadian experience. Neurosurgery 60:881-886, 2007

6. Hubbard ZS, Shah AH, Ragheb M, Wang S, Jernigan S, Ragheb J: Economic benefit of neurosurgical intervention for infant hydrocephalus in Haiti. J Neurosurg Pediatr 24:306314, 2019

7. Joseph FJ, Bernard J Jr, Augustin S Jr: Factors associated with postoperative complications in hydrocephalic infants diagnosed at Bernard Mevs Hospital in Port-au-Prince, Haiti, from 2011 to 2013. World Neurosurg 103:386-390, 2017

8. Kamran N, Calinescu A, Candolfi M, Chandran M, Mineharu Y, Asad AS, et al: Recent advances and future of immunotherapy for glioblastoma. Expert Opin Biol Ther 16:1245-1264, 2016

9. Kulkarni AV, Drake JM, Kestle JR, Mallucci CL, Sgouros S, Constantini S: Predicting who will benefit from endoscopic third ventriculostomy compared with shunt insertion in childhood hydrocephalus using the ETV Success Score. J Neurosurg Pediatr 6:310-315, 2010

10. Kulkarni AV, Riva-Cambrin J, Browd SR, Drake JM, Holubkov R, Kestle JR, et al: Endoscopic third ventriculostomy and choroid plexus cauterization in infants with hydrocephalus: a retrospective Hydrocephalus Clinical Research Network study. J Neurosurg Pediatr 14:224-229, 2014

11. Kulkarni AV, Schiff SJ, Mbabazi-Kabachelor E, Mugamba J, Ssenyonga P, Donnelly R, et al: Endoscopic treatment versus shunting for infant hydrocephalus in Uganda. N Engl J Med 377:2456-2464, 2017

12. Kulkarni AV, Warf BC, Drake JM, Mallucci CL, Sgouros S, Constantini S: Surgery for hydrocephalus in sub-Saharan Africa versus developed nations: a risk-adjusted comparison of outcome. Childs Nerv Syst 26:1711-1717, 2010

13. Oakes WJ: Shunts in Africa. J Neurosurg 102 (4 Suppl):357, 2005

14. Ragheb M, Shah AH, Jernigan S, Koru-Sengul T, Ragheb J: Epidemiology of pediatric hydrocephalus in Haiti: analysis of a surgical case series. J Neurosurg Pediatr 23:568-576, 2019
15. Salvador SF, Oliveira J, Pereira J, Barros H, Vaz R: Endoscopic third ventriculostomy in the management of hydrocephalus: outcome analysis of 168 consecutive procedures. Clin Neurol Neurosurg 126:130-136, 2014

16. Shah AH, Barthélemy E, Lafortune Y, Gernsback J, Henry A, Green B, et al: Bridging the gap: creating a self-sustaining neurosurgical residency program in Haiti. Neurosurg Focus 45(4):E4, 2018

17. Warf BC: Comparison of 1-year outcomes for the Chhabra and Codman-Hakim Micro Precision shunt systems in Uganda: a prospective study in 195 children. J Neurosurg 102 (4 Suppl):358-362, 2005

18. Warf BC: Congenital idiopathic hydrocephalus of infancy: the results of treatment by endoscopic third ventriculostomy with or without choroid plexus cauterization and suggestions for how it works. Childs Nerv Syst 29:935-940, 2013

19. Warf BC: Hydrocephalus in Uganda: the predominance of infectious origin and primary management with endoscopic third ventriculostomy. J Neurosurg 102 (1 Suppl):1-15, 2005

20. Warf BC: Pediatric hydrocephalus in East Africa: prevalence, causes, treatments, and strategies for the future. World Neurosurg 73:296-300, 2010

21. Warf BC, Alkire BC, Bhai S, Hughes C, Schiff SJ, Vincent $\mathrm{JR}$, et al: Costs and benefits of neurosurgical intervention for infant hydrocephalus in sub-Saharan Africa. J Neurosurg Pediatr 8:509-521, 2011

22. Warf BC, Mugamba J, Kulkarni AV: Endoscopic third ventriculostomy in the treatment of childhood hydrocephalus in Uganda: report of a scoring system that predicts success. J Neurosurg Pediatr 5:143-148, 2010

23. Warf BC, Tracy S, Mugamba J: Long-term outcome for endoscopic third ventriculostomy alone or in combination with choroid plexus cauterization for congenital aqueductal stenosis in African infants. J Neurosurg Pediatr 10:108-111, 2012

24. Weil AG, Fallah A, Chamiraju P, Ragheb J, Bhatia S: Endoscopic third ventriculostomy and choroid plexus cauterization with a rigid neuroendoscope in infants with hydrocephalus. J Neurosurg Pediatr 17:163-173, 2016

25. Zandian A, Haffner M, Johnson J, Rozzelle CJ, Tubbs RS, Loukas M: Endoscopic third ventriculostomy with/without choroid plexus cauterization for hydrocephalus due to hemorrhage, infection, Dandy-Walker malformation, and neural tube defect: a meta-analysis. Childs Nerv Syst 30:571-578, 2014

\section{Disclosures}

The authors report no conflict of interest concerning the materials or methods used in this study or the findings specified in this paper.

\section{Author Contributions}

Conception and design: all authors. Acquisition of data: all authors. Analysis and interpretation of data: all authors. Drafting the article: all authors. Critically revising the article: all authors. Reviewed submitted version of manuscript: all authors. Approved the final version of the manuscript on behalf of all authors: Shah. Statistical analysis: Cajigas. Study supervision: J Ragheb.

\section{Correspondence}

John Ragheb: University of Miami Miller School of Medicine, Nicklaus Children's Hospital, Miami, FL. jragheb@med.miami. edu. 